\title{
The Simple Enterprise Architecture Framework: Giving Alignment to IT Decisions
}

\author{
Giovanni Giachetti ${ }^{1}$, Beatriz Marín ${ }^{2}$, and Estefanía Serral ${ }^{3}$ \\ ${ }^{1}$ Universidad Tecnológica de Chile INACAP, Chile \\ ${ }^{2}$ Facultad de Ingeniería y Ciencias, Universidad Diego Portales, Chile \\ ${ }^{3}$ Faculty of Economics and Business, KU Leuven, Belgium \\ ggiachetti@inacap.cl, beatriz.marin@mail.udp.cl, estefania.serralasensio@kuleuven.be
}

\begin{abstract}
Context: Enterprise Architecture seeks to align organizational objectives with decisions associated to people, processes, information and technology. Different frameworks have been proposed for designing an enterprise architecture, such as TOGAF or Zachman. However, defining an enterprise architecture following these frameworks is not an easy task since it requires the alignment of organizational needs with technological decisions. Goal: This paper presents a methodological framework, called Simple Enterprise Architecture (SEA), that eases the definition of an enterprise architecture and provides concrete proof of the alignment between IT decisions and organizational needs. Method: The SEA framework has been developed by integrating components from existing ones in order to generate a concrete process that guides analysts in the correct definition of an enterprise architecture. Results: This framework has been used in teaching system architecture master courses for 5 years with positive results.
\end{abstract}

Keywords: Enterprise Architecture, Information Technology, Framework, Teaching, Lessons Learned, IT decisions.

\section{Introduction}

Enterprise Architecture (EA) is a field intended to improve the management and functioning of complex enterprises and their information systems by aligning organizational objectives with decisions associated to people, processes, information and technology. EA helps an organization to transform its business vision and strategy into effective enterprise change through a clear understanding of its current state (asis) and its expected future state (to-be) [13].

Numerous frameworks have been created for defining EA [20], such as the Open Group Architecture Framework (TOGAF) [7] or the Zachman framework [26]. They provide a set of practices or activities that guide the EA definition. However, correctly defining an EA using one of these frameworks (or combination of them) is still a complex task, since it requires understand each framework specification, its use to represent the set of key concepts related to the organizational context, and finally, understanding how to align the organizational needs with technological decisions that 
is represented by a conceptual specification. This complexity makes the quality of an EA modeling design directly dependent on the experience of the work team or external consultants that creates it.

In this paper, we present the Simple Enterprise Architecture (SEA) Framework that is oriented to guide analysts in the correct definition and validation of an enterprise architecture for IT projects. The SEA framework builds on and integrates different practices from the well-known EA frameworks TOGAF and the Zachman framework, with system architecture and measure specification methods, the Attribute-Driven Design [25] (ADD), the Architecture Tradeoff Analysis Method (ATAM) [12], and the Goal Question Metric (GQM) strategy [1]. Moreover, some lessons learned from the use of SEA framework in system architecture master courses for novel and senior engineers during five years are presented, which can be a reference for practitioners related to use and/or teaching of enterprise architectures.

The rest of the paper is organized as follows. Section 2 presents the state of the art and related work on EA architectures, Section 3 introduces the SEA framework. Section 4 presents the analysis and lessons learned from applying the SEA framework. Finally, Section 5 presents our main conclusions and future work.

\section{Related Work}

The initial idea about considering different dimensions of an enterprise was developed simultaneously within different disciplines in the early nineties. This inevitably led to the emergence of several EA frameworks (EAFs) [2]. An EAF tries to map the enterprise organizational goals to the work processes and IT infrastructure that are needed to reach those goals. EA frameworks support a broad range of objectives and enable decision making on different levels. According to [8][14], more than 50 EAFs are available to date. Some examples are: the Zachman framework, ARIS, TOGAF, the Federal Enterprise Architecture (FEA) framework, the Department of Defense Architecture Framework (DoDAF), the Treasury Enterprise Architecture Framework (TEAF), Enterprise Architecture Planning (EAP), or more recently, ArchiMate, and the integrated electronic Requirements Information Management framework (eRIM). For EAFs' comparisons and analyses, the authors refer to [2] [17] [14] [18] [27].

The Zachman Framework [26] is organized as a matrix with two dimensions. The first dimension represents six perspectives or views: Planner, Owner, Designer, Builder, Subcontractor, and User. The second dimension deals with the following six basic questions: what, how, where, who, when and why. These questions allow to gain insights into different aspects of an enterprise. The framework does not provide guidance on sequence, process, or implementation, but rather focuses on ensuring that all views are well established, regardless the order in which they are created.

ARIS [19] is a framework for modeling business information systems from a process-based perspective. The framework is close to a classic software development process consisting of the sequence of requirements elicitation, design specification, and implementation description, applied to the different views of a business information systems: organization, data, function, output, and control. 
TOGAF [7] provides a structure and classification schema used as a reference for architecture development. It gives a comprehensive description of the relevant elements of EA and provides a clear distinction of the business oriented description and the derived technological implementation. A key element of TOGAF is the Architecture Development Method (ADM), which is an iterative method for developing EA. TOGAF explains rules for developing three levels of principles: 1) support decision making across the entire enterprise, 2) provide guidance of IT resources, and 3) support architecture principles for development and implementation.

The FEA framework [11] consists of a set of interrelated reference models that provide standardized categorization for strategic, business, and technology models. The main goal of FEAF is to organize and promote sharing of Federal information for the entire Federal Government. FEA allows for flexibility in the use of methods, work products, and tools to be used by the individual federal agencies.

DoDAF [4] is a holistic framework and conceptual model for EA development particularly in Department of Defense agencies. The framework builds on three sets of views: Operational, System, and Technical Standards. These views are linked in a fourth view by means of a dictionary to define terms and by providing context, summary, or overview-level information. This framework provides descriptions of final products as well as guidance and rules for consistency to facilitate comparison and integration of systems and architectures.

TEAF [3] aims at facilitating the integration, information sharing, and exploitation of requirements across the Department of the Treasury [3]. Similar to DoDAF, TEAF includes descriptions of work products for documenting and modeling enterprise architectures. The framework was created to map the interrelationships among the organizations of the department in order to manage IT resources.

EAP [22] proposed by Capgemini contains activities and processes in order to achieve a To-Be architecture by considering four perspectives: Business, Data, Application, and Infrastructure.

The ArchiMate Standard [5] is a synthesis of ideas coming from previous EAFs. It introduces an integrated language for describing EAs. ArchiMate fits into the TOGAF framework as it provides concepts for creating a model that correlates to its three architectures (layers).

eRIM [10] was defined for managing information about client requirements across all phases of a construction project and through-life of a built facility. The framework defines an information-centric EA approach that is process and service-oriented.

Some works have also been proposed to integrate different EA frameworks in order to better support the design of an EA. The Integrated Architecture Framework (IAF) [24] is based on ideas from Zachman and EAP and it provides practical guidelines to apply it. In [15], a framework is presented for the definition of EA of government organizations. The framework integrates TOGAF ADM with the Service Architecture Network Architecture (SONA) for connecting network infrastructure, network services, and application. In [27], a framework that integrates Zachman, Four-domain (processes, procedures, business tools, and dependencies required to support business functions), TOGAF, and the Reference Model of Open Distributed Processing (RM-ODP) is introduced to be used as a common framework to communicate about EAFs of different businesses and relate them to each other. 
While some of these frameworks (e.g., DoDAF, FEAF, and TEAF) have been designed to address specific needs, others (e.g., Zachman and TOGAF) can be more widely applied. In any case, these frameworks are still too abstract, they lack of straightforward guidelines to apply them, which makes difficult their understanding and accurate application [23] [18]. Moreover, no framework provides a concrete process that guides analysts in the definition of an EA, which gives evidence on how IT decisions are aligned with the organizational goals. All these issues provoke that students cannot properly use these frameworks, resulting in a frustrating experience. Next section presents the SEA framework, which overcome the issues identified.

\section{The Simple Enterprise Architecture (SEA) Framework}

The SEA framework builds on the three levels shared by TOGAF and Zachman (see Fig. 1). The Business Architecture, shown on top of the pyramid, defines the business goals and processes, and is used as the driving artefact to align the two levels below: the Information Systems Architecture, which defines the design of the information systems that must support the defined business processes, and the Technical Architecture, which defines the technical infrastructure (e.g., hardware, networks, etc.) that will support those information systems.

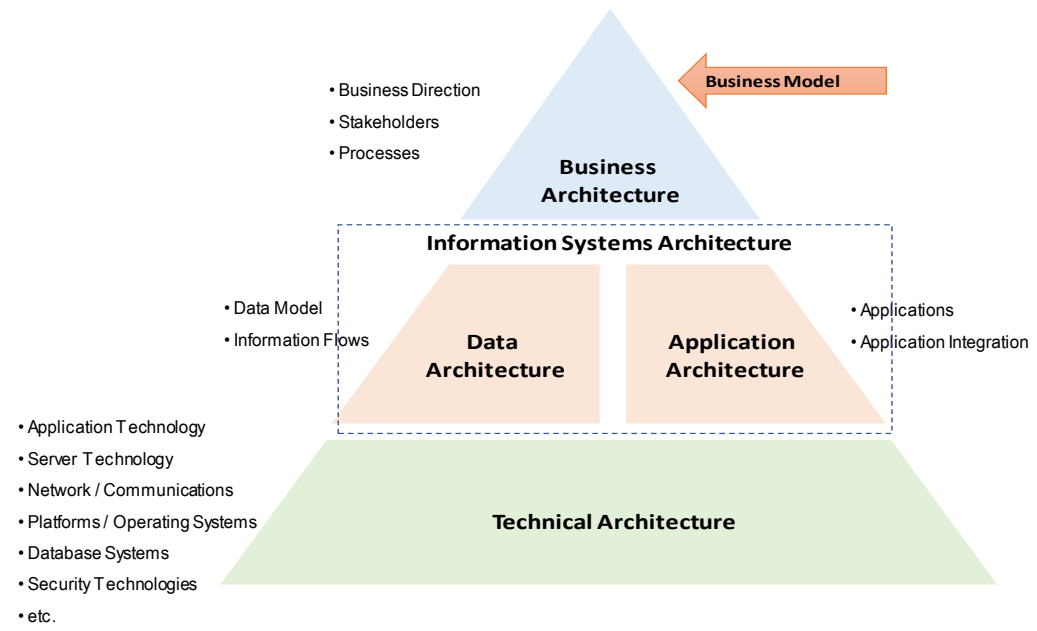

Fig. 1. Architectural Levels of the EAF Framework from TOGAF

To guide the alignment of these levels, quality attributes must be considered to evaluate to what extent the organizational requirements are met. These attributes are used to drive the information systems and technical decisions (at lower levels of the pyramid) that support the identified business processes. SEA applies the AttributeDriven Design [25] (ADD) method and the utility tree form Architecture Tradeoff Analysis Method (ATAM) to use these attributes as architectural drivers that guide analysts to refine the first version of the information system and technical 
architectures. Thus, the EA will be depicted in a way that all decisions of the three levels are aligned to these architectural drivers.

In order to validate and refine this version, we use tradeoffs analysis template from ATAM [12], which complements the previous step. This step allows obtaining a set of indicators that can be used to evaluate and support the architectural decisions.

Finally, the indicators obtained are used to define specific measures that demonstrate the alignment between the three levels, mainly the alignment of the technical decisions (technical architecture) with the business objectives. To obtain these measures, we use the GQM (goal, question, metric) approach.

Fig. 2 shows the complete SEA process. It is iterative and incremental, starting with a first definition of the EA guided by the business strategy. This definition is iteratively evaluated and refined by the application of ADD, ATAM and GQM.

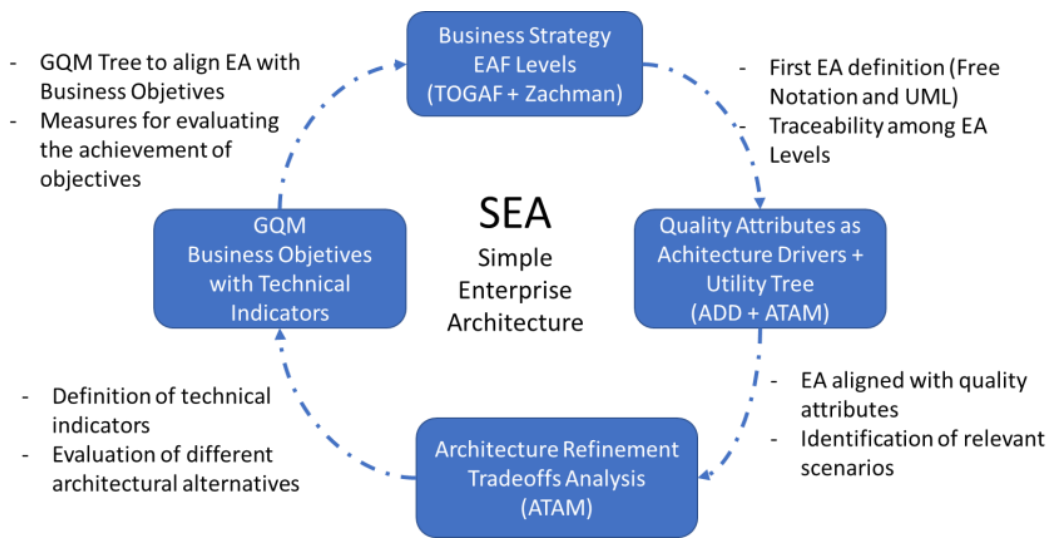

Fig. 2. Elements of the SEA Framework

Regarding the visual representation of the EA, we propose UML, a language that is very well known by IT analysts. We recommend using the use case diagram for business architecture, class model for data architecture, component (or package) model for application architecture, and the deployment model for the technical architecture. However, the correct use of the UML notation may also be an obstacle for defining the first version of the EA. Thus, we recommend using a free and very simple notation first, e.g., a notation based on boxes and arrows, and use the UML to give a detailed and refined EA definition once a first EA version is obtained.

\subsection{Business Strategy: EAF Levels}

The starting point of SEA is to stablish a first proposal for the EA taking as guidance the business strategy, by following the next steps: definition of the business architecture, definition of the data architecture, definition of the application architecture, and definition of the technical architecture.

The business architecture determines the data and application architecture, but also the implementation of new technology can modify the data and the business architectures: the business strategy and the technology of an organization are tightly related and greatly influence each other. Therefore, once the business, the data, and 
the application architectures are defined, they are refined starting the process in the opposite order (see bottom of Fig. 3): first considering the application architecture, then the data architecture, and finally the business architecture. In this second iteration, the first EA proposal is analyzed to evaluate whether additional elements are necessary. A new component in the application architecture can create the need to support additional data, and consequently the need of additional processes that support the management of those data. Normally, new elements for adding functionality or services not previously supported and needed to perform the organizational change are identified in this second iteration, such as applications to integrate legacy systems, to deal with security issues, or to support distributed services or user interfaces (Web, mobile, etc.).

The right-to-left process finishes when no new elements are identified. Once these three architectures are defined, a first definition of the technical architecture is created where the technical components to support the correct execution of the applications and data management are identified.

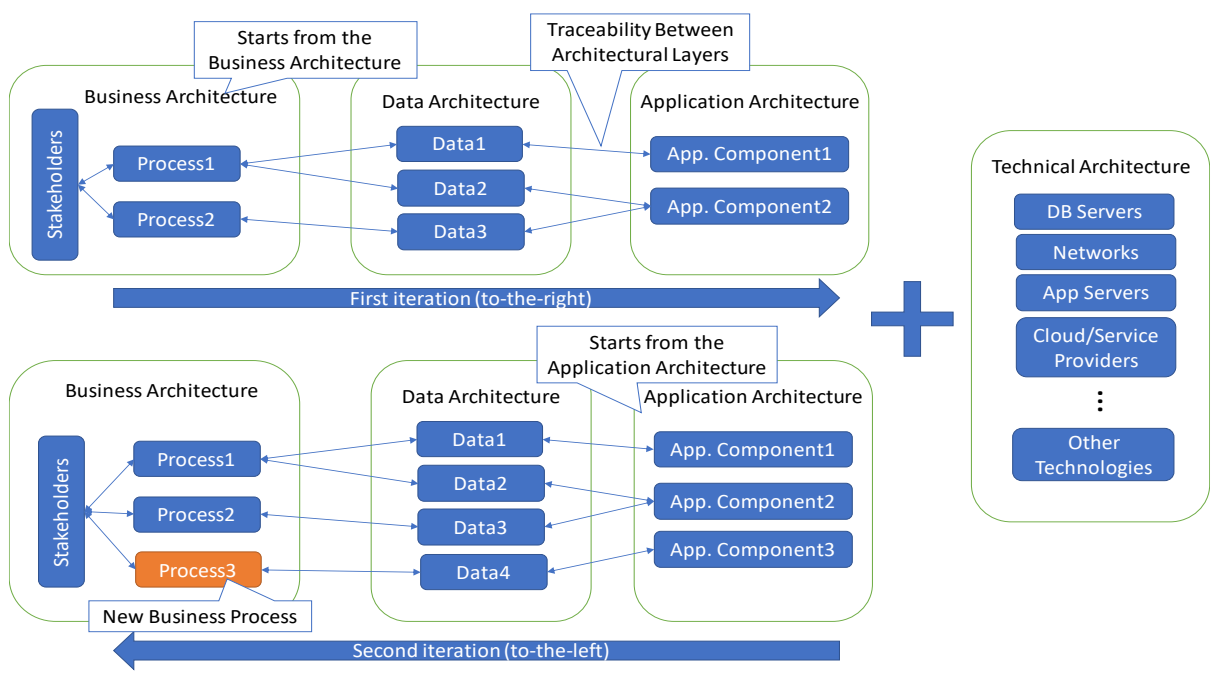

Fig. 3. Right-to-Left Definition Schema for EA Levels

3.2 Quality Attributes as Architectural Drivers

In order to organize the different architectural drivers, we extend the utility tree proposed by ATAM [12] (see Fig. 4). This tree identifies a set of quality attributes in the first level. These attributes are: Performance, Modifiability, Availability, and Security. In the second level of the tree, the different architectural drivers are detailed, and finally the intended scenarios for each driver are presented. In the SEA framework, we propose two changes to this utility tree. The first is to consider the six ISO 9126 [9] quality attributes and their sub-characteristics for internal and external quality instead of the four attributes originally proposed by ATAM.

As example, consider a Retail company that wants to develop a website for online sales. Important quality attributes in this example following the ISO 9126 classification are: security that comes from functionality and usability; and fault 
tolerance that comes from reliability. The following architectural drivers can be identified: payment security, ease of use, response time, and fault recovery.

ADD is applied to use quality attributes as architectural drivers. a) for each driver, specific scenarios are described to make explicit how the system should behave to successfully support the driver related to some quality attribute (third level of ATAM utility tree); b) one or more technical solutions (patterns) are proposed to deal with each scenario. To show the technical alternatives (patterns) related to the different scenarios, we have included an extra level after the scenarios definition in the utility tree of the ATAM approach (see Fig. 4). It is important to mention that a same pattern may support more than one scenario.

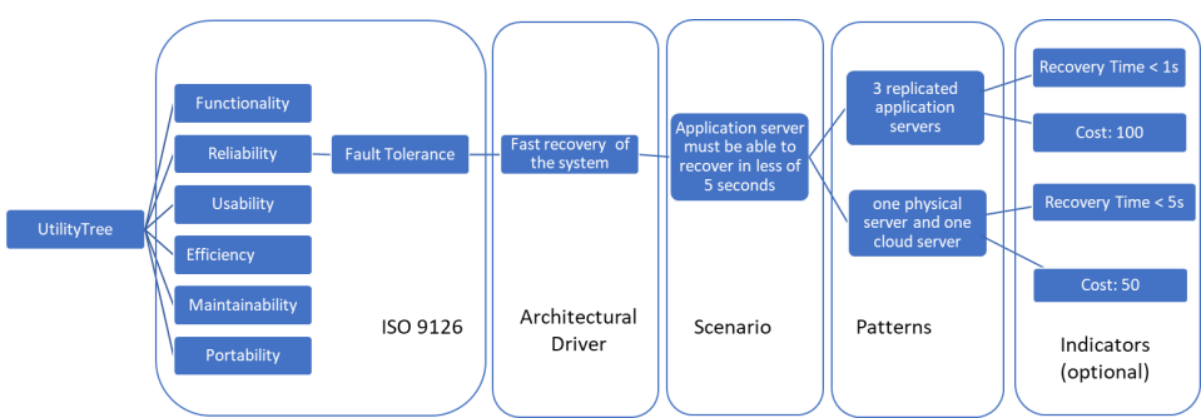

Fig. 4. SEA Utility Tree Schema (Reliability branch is detailed)

3.3 Tradeoff Analysis and Technical Indicators

In order to choose the appropriate patterns for the different scenarios, the SEA framework uses the tradeoffs analysis template proposed by ATAM [12]. For instance, from the information of Table1, if the cost of having the server down for 1 minute on Black Fridays is 500 then, the most appropriate pattern will be the 3 replicated servers. However, the architects can also choose to combine patterns, in case it is possible. For instance, another alternative is to consider the three replicated servers together with the cloud server since the cost having the server down is much higher and the cloud server can mitigate the risk R1 for pattern P1.

Table 1. Example of ATAM template for defining scenarios.

\begin{tabular}{|c|c|c|c|c|}
\hline \multicolumn{5}{|c|}{ Architectural Driver: Fast recovery of the system } \\
\hline \multicolumn{5}{|c|}{ Scenario: Application server must be able to recover in less of 5 minutes } \\
\hline \multicolumn{5}{|c|}{ Environment: Execution with more than 10.000 concurrent customers (Black Friday) } \\
\hline \multicolumn{5}{|c|}{ Stimulus: The main server is not responding } \\
\hline \multicolumn{5}{|c|}{ Response: The system resumes in less of 5 minutes } \\
\hline Achitectural Patterns & Sensitivity & TradeOff & Risk & No Risk \\
\hline P1: 3 replicated servers & & T1 & R1 & \\
\hline P2: local and cloud server & S1 & $\mathrm{T} 2$ & & \\
\hline Reasoning & \multicolumn{4}{|c|}{$\begin{array}{l}\text { T1: Lower recovery time ( } 1 \mathrm{~min}) \text {, but higher cost }(100) \\
\text { T2: Lower cost }(50) \text {, but higher recovery time }(5 \mathrm{~min}) \\
\text { S1: Dependency of the cloud provider } \\
\text { R1: The three servers may fail }\end{array}$} \\
\hline
\end{tabular}


3.4 Measures for Aligning Technical Architecture and Strategy.

In order to make explicit the relation between the technical architecture indicators and the organizational goals, we use the GQM approach. GQM allows us to generate measures that use these indicators as input GQM proposes the following three levels to align objectives with specific measures: Conceptual Level - Goals, Operational Level- Questions, and Quantitative Level - Measures.

At the conceptual level, the goals that have driven the EA are identified. In the Retail example, to increase the number of sales is a goal. At the operation level, we define the questions to evaluate to what extent the goal is reached. A key aspect to increase sales is to offer a good customer service. Thus, a question associated to this goal can be, e.g., is the company offering a good customer service? Questions can be linked to more than one goal, e.g., knowing if a company offers a good customer service is also important for the goal of increasing customer satisfaction.

Finally, the quantitative level set the measures that can evaluate the questions in a quantitative manner. Fig. 5 shows that the Recovery time indicator enables the definition of the measures cost of system down and number of unattended requests. This indicator is obtained from the tradeoffs analysis in the previous (ATAM) step.

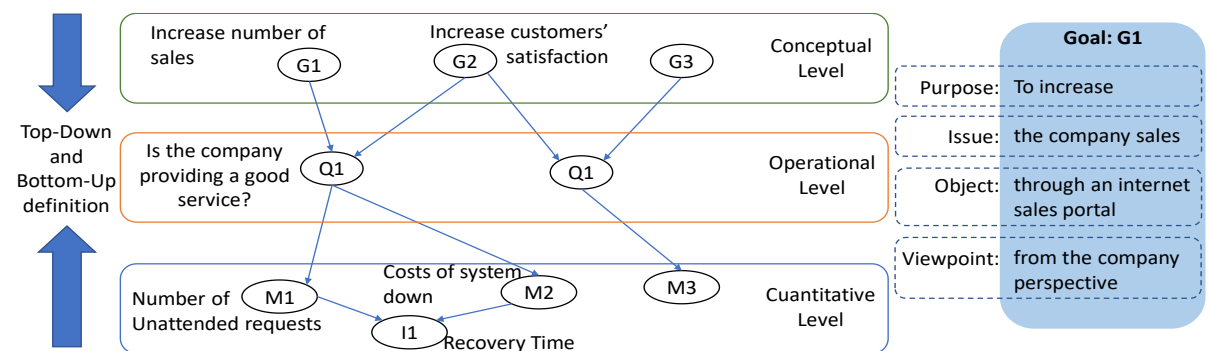

Fig. $5 \mathrm{GQM}$ for generation of measures aligned with business objectives

We recommend applying GQM by following a hybrid approach (between topdown and bottom-up). Fig 5 shows the indicator I1 (recovery time), which allows the definition of the measure number of unattended request and costs of system down. In this case, the lower is the recovery time, the lower is the cost to deal with the system failure as well as the not processed requests.

\section{Analysis and Lessons Learned}

Previous to the use of SEA framework, former students of the system architecture master course fail in the proper alignment of the IT architecture with the organizational goals, in the representation of the architectural models, and finally, they were not able to explain how the defined architecture fits to the organizational needs. Moreover, we have observed that students put the main focus on the intended system functionality, omitting how the system interacts with other organizational processes and stakeholders.

During the last five years, SEA has been used by more than 400 students of the system architecture course of a master program. The SEA framework breaks the lack 
of organizational vision, providing a systematic process and tools that guide the students in the definition of technical solutions driven by the business strategy.

The use of a well-known notation as UML facilitates the communication among the technical team. However, it can be difficult to start with the architecture representation in a specific notation since students pay more attention to properly represent the model more than to determine if all the relevant elements are included. To solve this issue, we propose to start with a free notation (boxes and arrows) for representing the initial EA model, mainly centered on identifying the relevant concepts, and, in a further step, to translate the initial model with a concrete notation.

We have observed another interesting effect of translating the EA from a free notation model to a concrete notation model: inconsistencies are identified during the translation process. Normally, these inconsistencies are non-identified actors (stakeholders) or hidden uses cases (process). Thus, this translation step also works as a verification activity for the EA specification using SEA.

\section{Conclusions and Future Work}

This paper has presented a simple framework called SEA that integrates best practices from well-known EAFs and system architecture and measure specification methods. The SEA framework has been designed to systematically guide analysts in the correct definition of EAs. The SEA framework facilitate the proper design of technical solutions, and also provides evidence that allows these solutions to be evaluated in terms of how they contribute to the achievement of organizational goals.

The aim of the SEA framework is to facilitate the teaching of EA courses at computer engineering careers, due to existing approaches, such as [16], where too complex according to the students perception. The evaluations surveys of the courses where SEA was applied has scores of $90 \%$ or above regarding comprehension, support and guidance material, ability of apply the knowledge acquired, and the feasibility of use SEA in professional practice. In contrast, similar curses without SEA have an average between $50-70 \%$. This provides an initial evidence of the perception of the students about the use of SEA framework to correctly define an enterprise architecture. Nevertheless, the validation of the SEA framework and industrial empirical evaluation of SEA is planned as future work.

Finally, we are preparing an online course to teach the SEA framework and facilitate its free use by practitioners and students, this is the Open SEA initiative. Some online lectures have already been recorded (such as https://vimeo.com/album/2472336/video/72053189).

\section{References}

1. Basili, V., Caldeira, G., Rombach, H.D.: The Goal Question Metric Approach, Wiley (1994),

2. Buckl, S., Schweda, C.M.: On the state-of-the-art in enterprise architecture management literature, Technische Universität München, Munich (2011),

3. Goethals, F.: An Overview of Enterprise Architecture Framework Deliverables (2003), 
4. Group, D.o.D.A.F.W.: Department of Defense Architecture Framework. January (2003)

5. Group, T.O.: The Open Group, ArchiMate 2.0 Specification, Berkshire, UK (2012),

6. Habra, N., Abran, A., Lopez, M., Sellami, A.: A framework for the design and verification of software measurement methods. Journal of Systems and Software, vol. $81 \mathrm{n}^{\circ}$ 5, 633-648 (2008) 7. Haren, V.: TOGAF Version 9.1, isbn: 9087536798. Van Haren Publishing (2011)

8. Hinkelmann, K., Gerber, A., Karagiannis, D., Thoenssen, B., Van der Merwe, A., Woitsch, R.: A new paradigm for the continuous alignment of business and IT: Combining enterprise architecture modelling and enterprise ontology. Computers in Industry, vol. 79, 77-86 (2016)

9. ISO: ISO/IEC 9126-1. In, vol. Software engineering - Product quality - Part 1: Quality Model. ISO (2001)

10. Jallow, A.K., Demian, P., Anumba, C.J., Baldwin, A.N.: An enterprise architecture framework for electronic requirements information management. International Journal of Information Management, vol. $37 \mathrm{n}^{\mathrm{o}}$ 5, 455-472 (2017)

11. Ji, W.-1., Xia, A.-b.: Federal enterprise architecture framework. COMPUTER INTEGRATED MANUFACTURING SYSTEMS-BEIJING-, vol. 13 nº 1, 57 (2007)

12. Kazman, R., Klein, M., Clements, P.: ATAM: Method for architecture evaluation, Carnegie-Mellon Univ Pittsburgh PA Software Engineering Inst (2000),

13. Lapkin, A., Allega, P., Burke, B., Burton, B., Bittler, R.S., Handler, R.A., James, G.A., Robertson, B., Newman, D., Weiss, D.: Gartner clarifies the definition of the term'enterprise architecture'. Gartner research, (2008)

14. Matthes, D.: Enterprise Architecture Frameworks Kompendium: Über 50 Rahmenwerke für das IT-Management. Springer-Verlag (2011)

15. Muhamad-Firmansyah, C., Bandung, Y.: Designing an enterprise architecture government organization based on TOGAF ADM and SONA. In: 2016 Int. Conf. on Information Technology Systems and Innovation (ICITSI), pp. 1-6. IEEE (2016)

16. Pereira, C.M., Sousa, P.: A method to define an Enterprise Architecture using the Zachman Framework. ACM symposium on Applied computing. ACM (2004) 1366-1371

17. Romero, D.a.V., F.: Enterprise information systems state of the art: past, present and future trends. Computers in Industry, $\mathrm{n}^{\circ}$ 79, 3-13 (2016)

18. Rouhani, B.D., Mahrin, M.N.R., Nikpay, F., Najafabadi, M.K., Nikfard, P.: A Framework for Evaluation of Enterprise Architecture Implementation Methodologies. Int. Journal of Social, Behavioral, Educational Economic, Business and Industrial Engineering, vol. $9 \mathrm{n}^{\circ} 1$ (2015)

19. Scheer, A.-W., Nüttgens, M.: ARIS architecture and reference models for business process management. Business Process Management, 301-304 (2000)

20. Schekkerman, J.: How to survive in the jungle of enterprise architecture frameworks: Creating or choosing an enterprise architecture framework. Trafford Publishing (2004)

21. Serral, E., De Smedt, J., Snoeck, M., Vanthienen, J.: Context-adaptive Petri Nets: Supporting adaptation for the execution context. Expert Systems with Applications, vol. $42 \mathrm{n}^{\circ}$ 23, 9307-9317 (2015)

22. Spewak, S.H., Tiemann, M.: Updating the enterprise architecture planning model. Journal of Enterprise Architecture, vol. $2 \mathrm{n}^{\circ}$ 2, 11-19 (2006)

23. Urbaczewski, L., Mrdalj, S.: A comparison of enterprise architecture frameworks. Issues in Information Systems, vol. $7 \mathrm{n}^{\circ}$ 2, 18-23 (2006)

24. Van't Wout, J., Waage, M., Hartman, H., Stahlecker, M., Hofman, A.: The integrated architecture framework explained: why, what, how. Springer Science \& Business Media (2010) 25. Wojcik, R., Bachmann, F., Bass, L., Clements, P., Merson, P., Nord, R., Wood, B.: Attribute-driven design (ADD), version 2.0, CARNEGIE-MELLON UNIV PITTSBURGH PA SOFTWARE ENGINEERING INST (2006),

26. Zachman, J.A.: Concepts of the framework for enterprise architecture, (1996),

27. Zarvić, N., Wieringa, R.: An integrated enterprise architecture framework for business-IT alignment. Designing Enterprise Architecture Frameworks: Integrating Business Processes with IT Infrastructure, vol. 63 (2014) 